\title{
OPTICAL COUNTERPART OF ICRF: HIPPARCOS
}

\author{
L. LINDEGREN \\ Lund Observatory \\ Box 43, SE-22100 Lund, Sweden \\ AND \\ M.A.C. PERRYMAN \\ Astrophysics Division, ESTEC \\ 2200AG Noordwijk, The Netherlands
}

\section{The Hipparcos Catalogue}

The Hipparcos reference frame is defined by the positions and proper motions of $\sim 10^{5}$ stars given in the Hipparcos Catalogue (ESA, 1997). It is the optical counterpart of the International Celestial Reference Frame defined by the radio positions of extragalactic sources. The linking of the Hipparcos frame to the extragalactic frame is described by Kovalevsky et al. (1997) and Kovalevsky (1997). In the present review we consider only the intrinsic properties of the Hipparcos reference frame.

A complete description of the contents of the Hipparcos Catalogue is contained in Volume 1 of ESA (1997). Recent overviews have been given by Perryman et al. (1997) and Perryman (1997). The principal astrometric data provided are the barycentric coordinates $(\alpha, \delta)$ of the stars, the trigonometric parallaxes $(\pi)$ and the proper motion components $\left(\mu_{\alpha *} \equiv \mu_{\alpha} \cos \delta, \mu_{\delta}\right)$. These parameters are given in the main catalogue for 117955 entries along with estimated standard errors and correlation coefficients, all data being referred to the common reference epoch $T_{0}=J 1991.25$ on the Terrestrial Time (TT) scale. Typical ranges of the astrometric standard errors and some other characteristics of the catalogue are shown in Table 1.

The main astrometric data were obtained through a rigorous combination of the results of two independent analyses of the satellite observations, performed by the FAST and NDAC consortia. The parallel reduction scheme included a comprehensive system of cross-checking and validation at several levels of the processing to ensure the highest quality of the end product. The standard errors given in the catalogue are believed to be reliable, and generally representative of the true (external) errors to within a factor 1.0-1.2. Nevertheless, the short duration of the mission-compared with the time base of ground-based astrometric catalogues - and other specific features of the observations make it necessary to interpret the data with due respect for the potential complications caused, in particular, by double and multiple stars (Section 2.3). Flags and pointers in the main catalogue give additional indications of the quality of the solutions and may refer the user to the various annexes for details on specific objects.

TABLE 1. Main characteristics of the Hipparcos Catalogue. The $V$ magnitude and standard errors (s.e.) are median values for the whole catalogue, with the 10 th and 90 th percentiles given in parentheses.

\begin{tabular}{llll}
\hline Measurement period & $1989.85-1993.21$ & Magnitude $V$ & $8.44(6.76 / 9.88) \mathrm{mag}$ \\
Catalogue epoch $T_{0}$ & $\mathrm{~J} 1991.25(\mathrm{TT})$ & S.e. in $\alpha *$ at $T_{0}$ & $0.87(0.53 / 1.70) \mathrm{mas}$ \\
Nominal reference system & ICRS & S.e. in $\delta$ at $T_{0}$ & $0.72(0.51 / 1.38) \mathrm{mas}$ \\
coincidence with ICRS at $T_{0}$ & $\pm 0.6 \mathrm{mas}$ & S.e. in $\pi$ & $1.10(0.71 / 1.91) \mathrm{mas}$ \\
$\quad$ residual rotation wrt ICRS & $\pm 0.25 \mathrm{mas} / \mathrm{yr}$ & S.e. in $\mu_{\sigma_{*}}$ & $1.02(0.60 / 1.98) \mathrm{mas} / \mathrm{yr}$ \\
Number of entries & 118218 & S.e. in $\mu_{\delta}$ & $0.85(0.57 / 1.56) \mathrm{mas} / \mathrm{yr}$ \\
with associated astrometry & 117955 & Ratio external error $/$ s.e. & $\sim 1.0-1.2$ \\
with associated photometry & 118204 & Level of systematic errors $<0.1 \mathrm{mas}$ \\
\hline
\end{tabular}




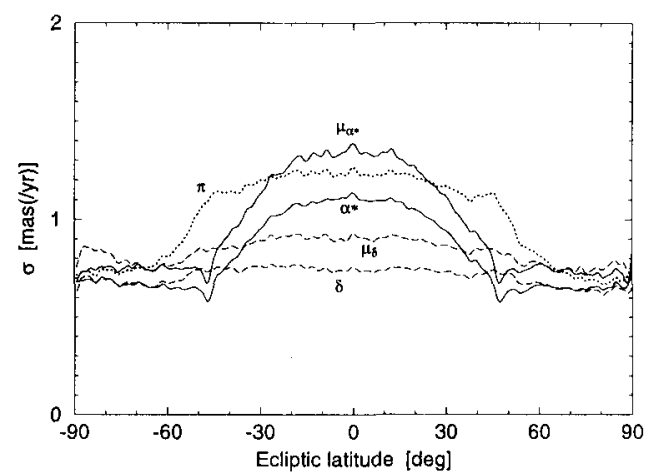

Figure 1. Median standard errors of the astrometric parameters (epoch J1991.25) as function of ecliptic latitude.

The five astrometric parameters $\alpha, \delta, \pi, \mu_{\alpha *}, \mu_{\delta}$ together with the (astrometrically undetermined) radial velocity component define the rectilinear space motions of the stars relative to the solar system barycentre. To within observational uncertainties, this standard model applies to single stars as well as to several categories of non-single stars, such as the components of wide pairs with long orbital periods and the photocentres of close binaries whose absolute orbits have long periods or small amplitudes. The standard model was adopted for 100038 catalogue entries regarded as single stars, and for the resolved components of 13211 additional entries, with details given in the Double and Multiple Systems Annex C. Of the remaining 4706 entries with accepted astrometric solutions, most are astrometric binaries with significantly curved proper motion or even complete orbital solutions for the motion of the photocentre (Lindegren et al. 1997). For these objects the astrometric parameters given in the main catalogue refer to the mean motion over the $\sim 3$ year measurement period, or (for orbital solutions) to the motion of the centre of mass of the system. Parts G, O, V and X of the Double and Multiple Systems Annex give details of these solutions.

\section{Properties of the Hipparcos Reference Frame}

In this section we review some of the unusual characteristics of the Hipparcos reference frame and address the important question of the systematic accuracy of the data.

\subsection{ACCURACY VERSUS POSITION ON THE SKY}

From ground-based astrometry we are used to regard the measurements in $\alpha$ and $\delta$ as virtually independent, sometimes produced by different instruments, and usually affected by different kinds of errors. With Hipparcos all measurements are one-dimensional, produced by a single instrument scanning the sky in a complex pattern. Moreover, the scanning law is quasi-symmetric with respect to the ecliptic so that the main variation of the standard errors over the sky is a function of ecliptic latitude (Figure 1), and the principal axes of the error ellipses are on the average oriented along the ecliptic coordinates. As a consequence of the scanning law the distribution of errors is distinctly different in the various regions depending on the ecliptic latitude $\beta$ :

- in the ecliptic region, $|\beta|<30^{\circ}$, the density of observations was rather low, with scans predominantly in the north-south direction, leading to error ellipses elongated along the ecliptic;

- in two narrow bands around $|\beta| \simeq 47^{\circ}$ the density of observations was very high with scans predominantly in the east-west direction, leading to error ellipses slightly compressed along the ecliptic;

- in the polar regions, $|\beta|>60^{\circ}$, the density was moderately high with a rather uniform coverage in different directions, leading to almost constant and isotropic errors.

This pattern is roughly the same for the proper motions as for the positions at the reference epoch, and it therefore persists also when the positions are propagated to other epochs. 


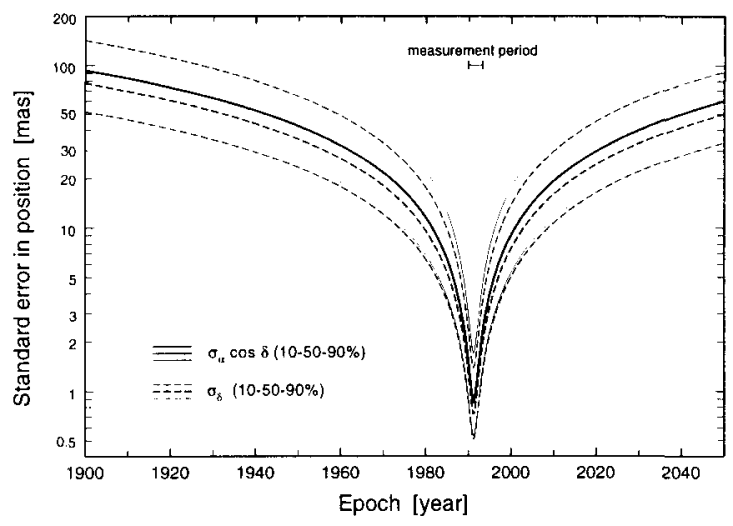

Figure 2. The accuracy of individual stellar positions at various epochs is here represented by the 10th, 50th and goth percentiles of the standard errors in right ascension and declination.

\subsection{POSITIONAL ERROR VERSUS TIME}

The positional reference frame is defined to sub-milliarcsec accuracy only within a few years of the catalogue epoch J1991.25 and degrades rapidly with the time difference from that epoch (Figure 2). This is a direct consequence of the short measurement period of $\sim 3$ years. However, even after several decades the individual accuracies are at least as good as in the best ground-based optical catalogues. Thanks to the expected low level of systematic errors (Section 2.4) and the high density of stars, the statistically defined reference frame is likely to be superior to all existing ground-based optical frames independent of epoch. While the systematic character of the Hipparcos reference frame therefore cannot be improved by incorporating old catalogues, the accuracies of individual objects may in some cases be improved by such a process.

\subsection{ROLE OF ASTROMETRIC BINARIES}

An important consequence of the presence of astrometric binaries is that the quasi-instantaneous proper motion measured by Hipparcos may be significantly different from the long-term motion measured on ground. This is obvious in the cases where Hipparcos measured a significantly nonuniform motion (Double and Multiple Systems Annex G), but probably applies also to several thousand (as yet) unrecognised astrometric binaries, which in the Hipparcos data analysis appeared to be perfectly normal single stars. When the Hipparcos data are extrapolated to epochs several decades before or after the measurement period, the distribution of positional errors will have an extended, non-Gaussian tail largely consisting of such astrometric binaries. This effect was not taken into account in constructing Figure 2, as the statistics of the phenomenon are not well known. Aspects of the problem have been discussed by Wielen (1997).

\subsection{GLOBAL COHERENCE BY DESIGN}

Several key features of the Hipparcos mission were specifically aimed at providing an optical reference frame of unprecedented coherence on a global scale, i.e. a system of positions and proper motions free of internal distortion:

- observations from space virtually eliminated all effects of refraction, instrumental flexure and thermal distortion;

- all-sky visibility permitted a direct linking of the stars over the whole celestial sphere observed with a single instrument;

- the two viewing directions of the satellite, separated by a large and suitable angle, resulted in a 'rigid' connection of one-dimensional measurements in different parts of the sky; 


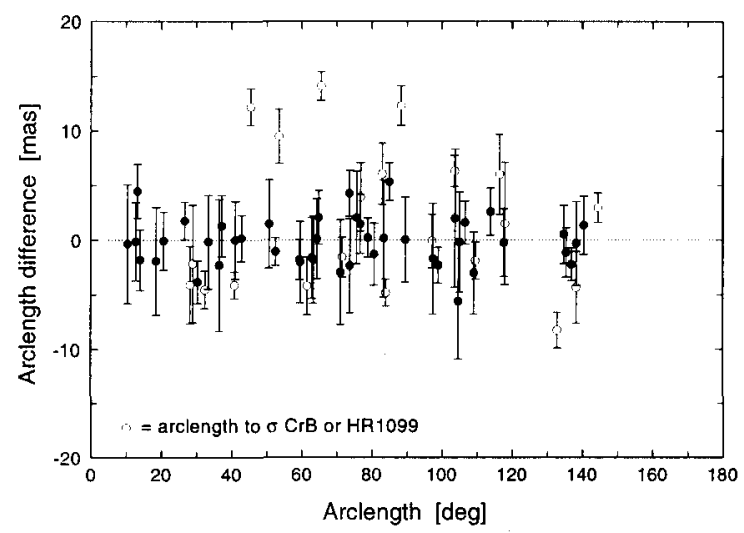

Figure 3. Differences in arclength between Hipparcos and VLBI observations of 12 radio stars. This is a test of the internal consistency of the Hipparcos reference frame. Apart from arclengths involving $\sigma \mathrm{Cr} B$ (HIP 79607) or HR 1099 (HIP 16846) the arclength differences are well centred with a scatter in perfect agreement with the combined standard errors of the Hipparcos and VLBI data.

- the continuous scanning of the satellite provided multi-epoch measurements in geometrical configurations favourable for the simultaneous determination of the five astrometric parameters of each star;

- critical geometrical calibrations of the instrument were included in a self-consistent manner in the global analysis of the measurements;

- the high redundancy factor of the observations (with typically $\sim 6$ independent measurements per astrometric parameter) provided high sensitivity to deviations from the standard model of uniform motion and allowed problematic objects to be diverted away from the main processing chain where they did not contribute to the frame definition.

The global analysis of the Hipparcos measurements was thus designed to provide a globally coherent reference frame, which was however decoupled from existing frames and a priori inertial directions. Its subsequent linking to the ICRF was considered a completely independent problem.

\subsection{TESTS OF GLOBAL COHERENCE}

The coherence of the Hipparcos reference frame can be tested by direct comparison with an independent reference frame of similar (or higher) accuracy, provided the catalogues have some sources in common. However, suitable comparison data are scarce, and for this reason one may also have to resort to indirect evidence. We describe briefly the results of one direct and four indirect tests.

Comparison with VLBI: 12 radio stars measured by VLBI as part of the extragalactic link programme (Kovalevsky et al. 1997) have positional accuracies at J1991.25 in the range from 0.3 mas to a few mas and may thus provide a stringent, if somewhat restricted test of the Hipparcos frame. In order to make the test independent of the frame orientation, arclengths were computed for all 66 possible object pairs both from the Hipparcos Catalogue and from the VLBI positions, and the arclength differences plotted against the arclength (Figure 3). The comparison shows a very good agreement between the Hipparcos and VLBI arclengths, on all angular scales, if allowance is made for a possible radio/optical offset in two of the objects.

FAST/NDAC comparisons: Although based on the same satellite data, the global reductions by the two consortia FAST and NDAC were to a large extent independent and used different models for the satellite motion, instrument calibrations, etc. Consistency between the consortia results was a necessary (but not sufficient) condition for global coherence of the combined catalogue. Extensive analyses of the FAST/NDAC differences did not reveal any large-scale systematic differences exceeding about 0.1 mas or 0.1 mas/yr.

Inclusion of bias parameters: It is possible to test for certain types of systematic errors by performing special solutions in which additional unknowns (bias parameters) are introduced. A priori, 
these parameters are expected to come out as zero, within their estimated standard errors. Tests of this kind are described in ESA (1997), Volume 3 (Section 20.4). The general conclusion was that systematic terms exceeding 0.1 mas are rare and affect mainly extreme cases such as very red stars $(V-I>2)$.

Parallax zero point: Of the five astrometric parameters, the parallax is normally the one most easily checked against independent information such as photometric or spectroscopic distances. This could be used to estimate the global zero-point error $(z)$ of the Hipparcos parallaxes and the ratio $(k)$ of the external to nominal parallax errors (Arenou et al. 1995). The results from several tests on different stellar samples indicated that $|z|<0.1$ mas and $k \simeq 1.05$. Since the (absolute) parallaxes and the positions and proper motions were obtained in the same global reduction, and the absoluteness of the parallaxes depends directly on the accuracy of the wide-angle measurements, it is difficult to imagine that significant large-scale distortion of the reference frame could result from the same measurements that appear to give such accurate parallaxes.

Determination of PPN parameter $\gamma$ : The bending of starlight in the solar gravitational field is proportional to $\frac{1}{2}(1+\gamma) \cot (\theta / 2)$, where $\gamma$ is the PPN parameter ( $=1$ in General Relativity) and $\theta$ is the angle between the star and the Sun. Hipparcos measured the light bending for $47^{\circ}<\theta<133^{\circ}$, where it amounts to 2-9 mas, and gave $\gamma=0.997 \pm 0.003$ (Frœeschlé et al. 1997). The uncertainty corresponds to a positional shift of 3 to $14 \mu \mathrm{as}$ at an angular scale length of the order of $90^{\circ}$. This suggests that the level of systematic errors in the Hipparcos measurements, at these scales, is very small indeed.

In summary the direct comparison with VLBI confirms global coherence on a level of about 1 mas. Several indirect tests based on many more stars suggest that the actual level of large-scale distortion is considerably smaller, i.e. of the order of 0.1 mas or less. This does not preclude a higher level of systematic errors in small areas of the sky or for extreme cases such as very red stars.

\section{Availability of Data}

The Hipparcos Catalogue is available as a 17-volume publication, ESA SP-1200. This includes the main Hipparcos Catalogue (as well as the Tycho Catalogue), the Double and Multiple Systems Annex, the Variability Annex, identification charts, light curves, a full sky star atlas, and extensive documentation of the contents of the catalogue and how it was constructed. All mission products, including intermediate astrometric and photometric information, are also provided on six ASCII CD-ROMs included within the 17-volume publication. On-line access to the catalogue data is provided through several services of the CDS, Strasbourg, and its mirror sites at ADC (Goddard) and NAO (Tokyo), and through the ESA Hipparcos WWW page.

\section{References}

Arenou, F., Lindegren L., Froeschlé, M., et al. (1995) Zero point and external errors of Hipparcos parallaxes, A\&A 304,52

ESA (1997) The Hipparcos and Tycho Catalogues, ESA SP 1200

Froeschle, M., Mignard, F., Arenou, F. (1997) Determination of the PPN parameter $\gamma$ with the Hipparcos data, Proc. HIPPARCOS Venice '97, ESA SP-402, p. 49

Kovalevsky, J. (1997) Linking Hipparcos to ICRF, this volume

Kovaleviky, J., Lindegren, L., Perryman, M.A.C., et al. (1997) The Hipparcos Catalogue as a realisation of the extragalactic reference system, A\&A 323,620

Lindegren, L., Mignard, F., Söderhjelm, S., et al. (1997) Double star data in the Hipparcos Catalogue, A\&A 323, L53

Perryman, M.A.C. (1997) The Hipparcos Catalogue contents, Proc. HIPPARCOS Venice '97, ESA SP-402, p. 1

Perryman, M.A.C., Lindegren, L., Kovalevsky, J., et al. (1997) The Hipparcos Catalogue, A\&A 323, L49

Wielen, R. (1997) Principles of statistical astrometry, A\&A 325, 367 